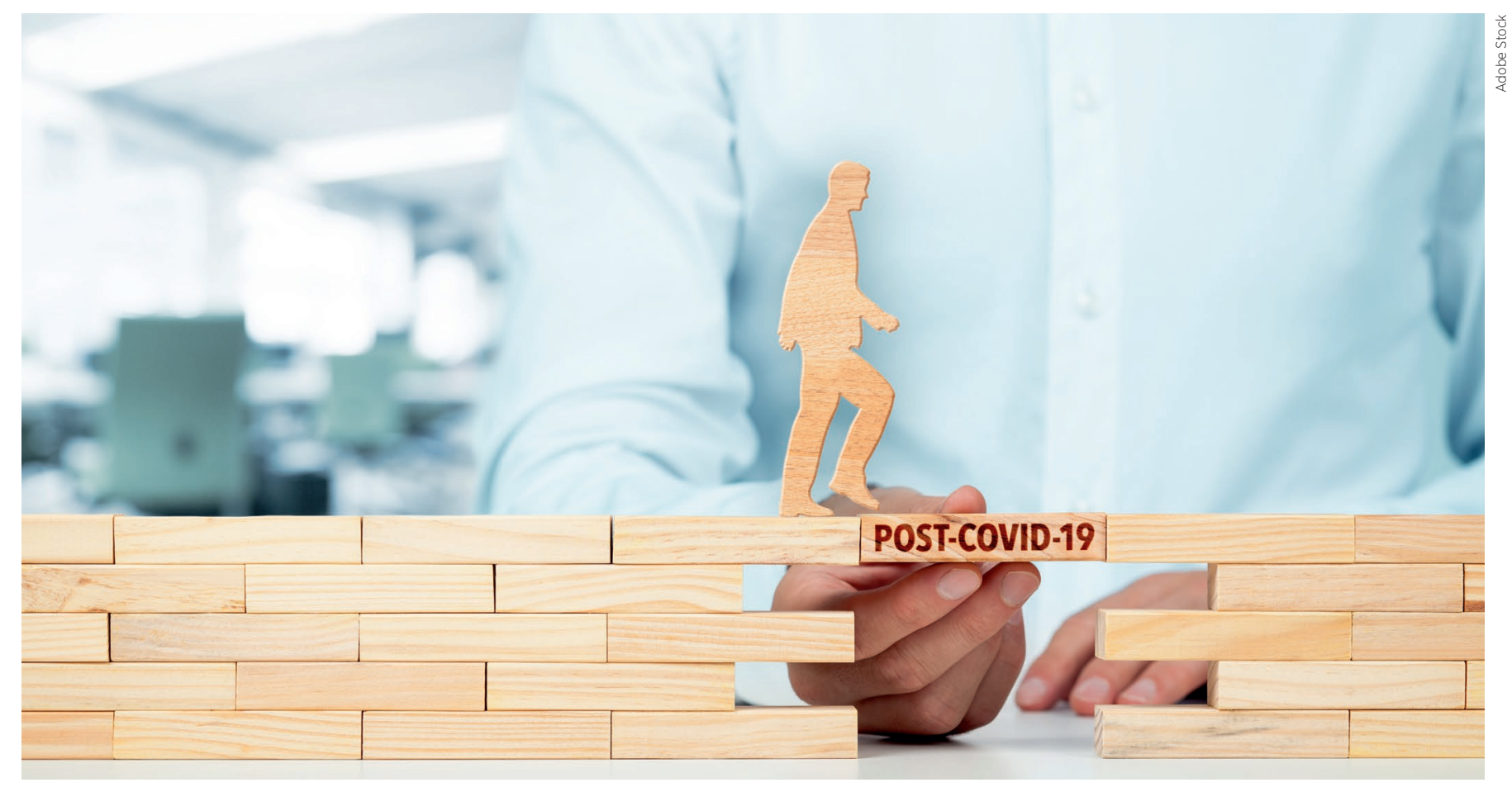

\title{
Post-COVID-Syndrom und die allgemeinen Auswirkungen - was sieht die Klinik?
}

Etwa zehn Prozent aller COVID-19-Patient*innen leiden unter Langzeitfolgen. ${ }^{1}$ Wie viele CoronaPatient*innen tatsächlich vom sogenannten Post-COVID-Syndrom bzw. von Long COVID betroffen sind, bedarf einer genauen Langzeiterhebung, an der es leider immer noch mangelt. Das deutsche Robert Koch-Institut „schätzt“ die Zahl der Genesenen und räumt ein, dass lang anhaltende Folgekrankheiten nach COVID-19 nicht statistisch erfasst werden².

s ist inzwischen folgende Stadien-
einteilung vorgenommen worden:

- Akute COVID-19-Erkrankung

- Multisystemisches inflammatorisches Syndrom (MIS, setzt zwei bis fünf Wochen nach Beginn der Infektion ein)

- Drittes Stadium (Wochen bis Monate nach der Infektion): Long COVID, Chronic COVID-19 (CCS) oder auch Post-COVID-Syndrom. Hier ist die Nomenklatur noch im Fluss. ${ }^{3}$

Seitens der Kliniker wird meistens der Begriff Post-COVID-Syndrom bevorzugt, was sich auch in der Einrichtung inzwischen zahlreicher „Post-COVIDAmbulanzen" niederschlägt. Sinnvoll wäre nach unserer Erfahrung die Differenzierung "Post-COVID-Syndrom" nach Verschwinden der Viren und "Long-COVID-19-Syndrom" bei wiederholtem Aufflackern der Infektion. Post-COVID kann auch nach soge-

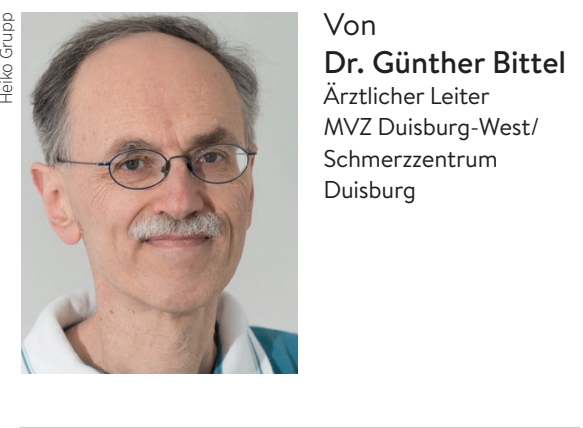

nannten „milden“ Verläufen entstehen. Verschiedene Verbände von Nervenärzt*innen und Psychotherapeut*innen stellen heute bereits gehäuft Depressionen, Angststörungen, Internetabhängigkeit und soziale Störungen vor allem bei Kindern und Jugendlichen fest - auch eine Folge des Versagens der Schul- und Jugendpolitik.

Zahlreiche chronische Schmerzpatient*innen, Angst- und Depressionspatient*innen werden durch das Wegfallen wichtiger Therapiemethoden wie Reha-
Sport, Wassergymnastik oder Gruppentherapie und durch soziale Isolierung und massive Ängste in ihrer Erkrankung weit zurückgeworfen. Die Forderung nach konsequentem Gesundheitsschutz bedeutet auch, Einschränkungen und Isolation so gering wie möglich zu halten. In einer Fragebogen-Untersuchung der Schmerzpatient*innen unseres Zentrums gaben 16 Prozent der Befragten an, dass sich ihr psychischer Zustand stark verschlechtert hat, 51 Prozent waren etwas verschlechtert. Bei 12 Prozent hatten sich die Schmerzen stark verschlechtert, bei 45 Prozent etwas, bei 40 Prozent waren sie gleich geblieben. Es gibt hier zwei Gefahren: Somatisierte Depressionen oder Somatisierungsstörungen werden zu Unrecht und mit Therapieversäumnissen als Post-COVID-Fälle abgelegt. Oder bei mangelhafter Diagnostik und Wissen über diese Zusammenhänge werden Post-COVID-Patient*innen häufig wie psychosomatisch oder primär psychisch 
Erkrankte behandelt und ihnen wird eine adäquate Therapie vorenthalten.

\section{HÄUFIG GEKLAGTE POST-COVID-BESCHWERDEN}

Oftmals stehen eine Konzentrationsschwäche, eine muskuläre Schwäche und eine allgemeine Leistungsminderung im Vordergrund. Häufig berichtet werden Müdigkeit, Atemnot und Schmerzen in der Brust. Bei Patient*innen nach $\mathrm{CO}$ VID-Pneumonie war in einer Studie die Diffusionskapazität für Kohlenmonoxid (DLCO) in vielen Fällen vermindert. Im Durchschnitt wurden 81 Prozent des normalen DLCO-Werts erreicht. Im CT wiesen 86 Prozent leichte Milchglastrübungen auf, 60 Prozent hatten Kerley-Linien und 26 Prozent radiologische Hinweise auf eine Lungenfibrose. ${ }^{4}$

Unter unseren eigenen Patient*innen mit Post-COVID-Symptomatik sehen wir bei einer Altersspanne von 17 bis 85 Jahren Belastungsdyspnoe, Herzjagen, rasche Erschöpfung bei ca. der Hälfte. Auch Patient*innen mit Infektion im Frühjahr 2020 berichten noch heute von lang anhaltenden Kopfschmerzen oder davon, dass sich der Geruchs- oder Geschmackssinn bisher nicht normalisiert hat.

Bislang ist die Studienlage zu den Langzeitfolgen einer Corona-Infektion noch unbefriedigend. Unterschiedliche Ergebnisse bringen Studien je nachdem, ob nur Menschen befragt oder untersucht wurden, die im Krankenhaus behandelt werden mussten, oder auch solche, die einen milden Verlauf hatten.

In England haben Forschende der staatlichen Gesundheitsbehörden über eine App die zahlreichen Symptome von Infizierten über Wochen und Monate untersucht - darunter fallen Kopfschmerzen, Müdigkeit, Gliederschmerzen, Atemprobleme, Gedächtnisverlust oder Konzentrationsstörungen. Demnach berichteten zehn Prozent der Teilnehmer*innen nach drei Wochen noch über verschiedenste Symptome, fünf Prozent nach fünf Wochen und zwei Prozent nach mehr als neun Wochen.

Martina Lenzen-Schulte zitiert im Deutschen Ärzteblatt eine aussagekräftige britische Studie: „Zu den wichtigen Arbeiten, die sich mit den eher schwer- wiegenderen Verläufen nach einem Klinikaufenthalt beschäftigen, zählt eine britische, die immerhin 384 stationär behandelte Patienten (Durchschnittsalter 59,9 Jahre, alle positiv auf SARSCoV-2 getestet) nachuntersucht hat. Aus dieser geht hervor, dass 8 Wochen nach der Entlassung noch 69 Prozent unter Fatigue und 53 Prozent unter Atemnot litten. Noch ein gutes Drittel (34\%) klagte über Husten und 14,6 Prozent hatten eine Depression 5 . In Sachen Laborwerte waren bei fast einem Drittel $(30,1 \%)$ die D-Dimere noch erhöht, fast jeder 10. (9,5\%) wies ein erhöhtes C-Reaktives Protein (CRP) auf. Hinzu kamen bei 38 Prozent pathologische Röntgenbefunde, darunter ein Viertel, das sich verschlechterte." ${ }^{\text {3 }}$

Hieraus lässt sich die berechtigte Forderung ableiten, dass diese Untergruppe noch drei Monate mit Gerinnungshemmern behandelt werden muss $(\mathrm{He}$ parine oder NOAKs).

Von mehr als 95.000 Patient*innen, die in England im Krankenhaus behandelt wurden (Stand März 2021), müsste nach Einschätzung der Gesundheitsbehörden fast die Hälfte auch nach der Entlassung medizinisch begleitet werden, etwa mit Reha-Maßnahmen. Sie müssen den Atemrhythmus trainieren oder psychisch betreut werden. Manche Langzeitfolgen können wiederum zu akuten Gesundheitsproblemen führen: Eine Studie zeigte, dass auch nach der Entlassung schwere Gesundheitsprobleme auftreten können. Bis zu ein Drittel der COVID-Patient*innen kam binnen fünf Monaten wieder ins Krankenhaus, mehr als dreimal so häufig wie vergleichbare Nicht-COVID-Patient*innen.

\section{RISIKOFAKTOREN UND SCHMERZ- THERAPEUTISCHE RELEVANZ}

Oft genannt werden folgende Faktoren, die Langzeitfolgen wahrscheinlicher machen:

- Schwere Krankheitsverläufe (häufige und schwere Symptome zu Krankheitsbeginn)

- Vorerkrankungen (zum Beispiel Übergewicht)

- Weibliches Geschlecht

Nach unseren Erfahrungen gehören die chronische Schmerzerkrankung und psychische Vorerkrankungen ebenfalls dazu. Bei chronischen Schmerzpatient*innen konnten wir beobachten:

- Verschlechterung oder Erstmanifestation von Myalgien, myofaszialen Schmerzen, Kopfschmerzen

- Verschlechterung oder Erstmanifestation von Fatigue, Depression und Ängsten, CFS

- Verschlechterung oder Erstmanifestation von Polyneuropathien

- Verschlechterung der Trainierbarkeit durch kardiopulmonale Folgen

- Auftreten von Guillain-Barré-Syndrom (Einzelfälle)

\section{WEITERE GESUNDHEITSPROBLEME BEI POST-COVID}

- Irritation und Schwächung des Immunsystems

- Verschlechterung oder Erstmanifestation von Diabetes mellitus (Pankreasbefall nachgewiesen)

- Gehäuftes Auftreten von Schlaganfällen, Lungenembolien, Herzinfarkten (wahrscheinlich weil nicht lange genug Heparin gegeben wurde!)

Diese Pandemie, die noch lange nicht vorüber ist, und ihre internationalen, oft erschütternden Begleiterscheinungen verbinden sich mit anderen krisenhaften Erscheinungen und werfen die Frage auf, wie es gesellschaftlich weitergehen soll, auch im Gesundheitswesen. Gebraucht werden eine COVID19-Langzeit-Datenbank, Massentestung, schnelle Impfung weltweit, Veränderung am Arbeitsplatz, in Verkehr, Schulen, Kitas, bei Wohnverhältnissen, Umweltschutz und der Situation der Geflüchteten (siehe Corona-Ausbrüche in Lagern und Massenunterkünften). Wir sind alle herausgefordert!

\section{Referenzen:}

1 DGP. Long-COVID: Leitlinie zur Diagnostik und Therapie auf dem Weg. 18. März 2021 https:// idw-online.de/de/news765168

2 Bittel-Mast-Wagner. COVID-19 - neuartig, gefährlich, besiegbar S.59 ISBN 978-3-88021-582-5

3 Der lange Schatten von COVID-19. Deutsches Arrzteblatt 49/2020 S. A2416

4 https://www.aerzteblatt.de/nachrichten/118773/ Post-COVID-Syndrom-Viele-Patienten-haben-Einschraenkungen-der-Lungenfunktion, Deutsches Ärzteblatt 26.11.2020

5 Manal S, Barnett J, Brill SE, et al. ,Long-COVID': a cross-sectional study of persisting symptoms, biomarker and imaging abnormalities following hospitalisation for COVID-19. Thorax. 10 Nov 2020. doi: 10.1136/thoraxjnl-2020-215818. (last accessed on 23 Nov 2020)

6 https://www.quarks.de/gesundheit/medizin/lang zeitschaeden-von-covid-19-was-wir-wissen-undwas-nicht/ 